Volume 69, Number 2, Pages 1418-1427(2020)

DOI: $10.31801 /$ cfsuasmas.764635

ISSN 1303-5991 E-ISSN 2618-6470

Received by the editors: July 06, 2020; Accepted: September 21, 2020

\title{
ON PROXIMITY SPACES AND TOPOLOGICAL HYPER NEARRINGS
}

\author{
Somaye BORHANI-NEJAD and B. DAVVAZ \\ Department of Mathematics, Yazd University, Yazd, IRAN
}

\begin{abstract}
In 1934 the concept of algebraic hyperstructures was first introduced by a French mathematician, Marty. In a classical algebraic structure, the composition of two elements is an element, while in an algebraic hyperstructure, the result of this composition is a set. In this paper, we prove some results in topological hyper nearring. Then we present a proximity relation on an arbitrary hyper nearring and show that every hyper nearring with a topology that is induced by this proximity is a topological hyper nearring. In the following, we prove that every topological hyper nearring can be a proximity space.
\end{abstract}

\section{INTRODUCTION}

In 1934, the concept of hypergroups was first introduced by a French mathematician, Marty 22 . In the following, it was studied and extended by many researchers, namely, Corsini [3], Corsini and Leoreanu [4], Davvaz [6 8], Frenni 12], Koskas 20], Mittas [23, Vougiouklis, and others. The topological hyper nearring notion is defined and studied by Borhani and Davvaz in [2].

In the 1950's, Efremovic 10,11, a Russian mathematician, gave the definition of proximity space, which he called infinitesimal space in a series of his papers. He axiomatically characterized the proximity relation $A$ is near $B$ for subsets $A$ and $B$ of any set $X$. The set $X$, together with this relation, was called an infinitesimal (proximity) space. Defining the closure of a subset $A$ of $X$ to be the collection of all points of $X$ near $A$, Efremovic 1011 showed that a topology can be introduced in a proximity space.

In this paper, we study some remarks on topological hyper nearring, then we

2020 Mathematics Subject Classification. Primary 16Y99; Secondary 20N20.

Keywords and phrases. Hyper nearring, topological hyper nearring, complete part, proximity relation.

borhani.math@yahoo.com; davvaz@yazd.ac.ir-Corresponding author;

(D) 0000-0001-2345-6789; X0000-0003-1941-5372.

(C) 2020 Ankara University Communications Faculty of Sciences University of Ankara-Series A1 Mathematics and Statistics 
define a proximity relation on hyper nearring and, we will prove that every hyper nearring with a topology that is induced by this proximity is a topological hyper nearring. In the following, we show that every topological hyper nearring is a proximity space.

\section{Preliminaries}

In this section, we recall some basic classical definitions of topology from 21 and definitions related to hyperstructures that are used in what follows.

Definition 1. 6] A hyper nearring is an algebraic structure $(R,+, \cdot)$ which satisfies the following axioms: hold:

(1) $(R,+)$ is a quasi canonical hypergroup, i.e., in $(R,+)$ the following conditions

(i) $x+(y+z)=(x+y)+z$ for all $x, y, z \in R$;

(ii) There is $0 \in R$ such that $x+0=0+x=x$, for all $x \in R$;

(iii) For any $x \in R$ there exists one and only one $x^{\prime} \in R$ such that $0 \in x+x^{\prime}$ (we shall write $-x$ for $x^{\prime}$ and we call it the opposite of $x$ );

(iv) $z \in x+y$ implies $y \in-x+z$ and $x \in z-y$.

If $A$ and $B$ are two non-empty subsets of $R$ and $x \in R$, then we define:

$$
A+B=\bigcup_{\substack{a \in A \\ b \in B}} a+b, x+A=\{x\}+\text { Aand } A+x=A+\{x\} .
$$

(2) $(R, \cdot)$ is a semigroup respect to the multiplication, having 0 as a left absorbing element, i.e., $x \cdot 0=0$ for all $x \in R$. But, in general, $0 \cdot x \neq 0$ for some $x \in R$.

(3) The multiplication is left distributive with respect to the hyperoperation + , i.e., $x \cdot(y+z)=x \cdot y+x \cdot z$ for all $x, y, z \in R$.

Note that for all $x, y \in R$, we have $-(-x)=x, 0=-0,-(x+y)=-y-x$ and $x(-y)=-x y$. Let $R$ and $S$ be two hyper nearrings. The map $f: R \rightarrow S$ is called a homomorphism if for all $x, y \in R$, the following conditions hold: $f(x+y)=$ $f(x)+f(y), f(x \cdot y)=f(x) \cdot f(y)$ and $f(0)=0$. It is easy to see that if $f$ is a homomorphism, then $f(-x)=-f(x)$, for all $x \in R$. A nonempty subset $H$ of a hyper nearring $R$ is called a subhyper nearring if $(H,+)$ is a subhypergroup of $(R,+)$, i.e., (1) $a, b \in H$ implies $a+b \subseteq H$; (2) $a \in H$ implies $-a \in H$; and (3) $(H, \cdot)$ is a subsemigroup of $(R, \cdot)$. A subhypergroup $A$ of the hypergroup $(R,+)$ is called normal if for all $x \in R$, we have $x+A-x \subseteq A$. Let $H$ be a normal hyper $R$-subgroup of hyper nearrring $R$. In [14, Heidari et al. defined the relation

$$
x \sim y(\bmod H) \text { if and only if }(x-y) \cap H \neq \emptyset \text {, for all } x, y \in H \text {. }
$$

This relation is a regular equivalence relation on $R$. Let $\rho(x)$ be the equivalence class of the element $x \in H$ and denote the quotient set by $R / H$. Define the 
hyperoperation $\oplus$ and multiplication $\odot$ on $R / H$ by

$$
\begin{aligned}
& \rho(a) \oplus \rho(b)=\{\rho(c): c \in \rho(a)+\rho(b)\}, \\
& \rho(a) \odot \rho(b)=\rho(a \cdot b),
\end{aligned}
$$

for all $a, b \in R$. Let $(R,+, \cdot)$ be a hyper nearring and $\tau$ a topology on $R$. Then, we consider a topology $\tau^{*}$ on $\mathcal{P}^{*}(R)$ which is generated by $\mathcal{B}=\left\{S_{V}: V \in \tau\right\}$, where $S_{V}=\left\{U \in \mathcal{P}^{*}(R): U \subseteq V, U \in \tau\right\}, V \in \tau$. In the following we consider the product topology on $R \times R$ and the topology $\tau^{*}$ on $\mathcal{P}^{*}(R)[2]$.

Definition 2. 2 Let $(R,+, \cdot)$ be a hyper nearring and $(R, \tau)$ be a topological space. Then, the system $(R,+, \cdot, \tau)$ is called a topological hyper nearring if

(1) the mapping $(x, y) \mapsto x+y$, from $R \times R$ to $\mathcal{P}^{*}(R)$,

(2) the mapping $x \mapsto-x$, from $R$ to $R$,

(3) the mapping $(x, y) \mapsto x . y$, from $R \times R$ to $R$,

are continuous.

Example 1. 2 The hyper nearring $R=(\{0, a, b, c\},+, \cdot)$ defined as follows:

\begin{tabular}{c|cccc}
+ & 0 & $a$ & $b$ & $c$ \\
\hline 0 & $\{0\}$ & $\{a\}$ & $\{b\}$ & $\{c\}$ \\
$a$ & $\{a\}$ & $\{0, a\}$ & $\{b\}$ & $\{c\}$ \\
$b$ & $\{b\}$ & $\{b\}$ & $\{0, a, c\}$ & $\{b, c\}$ \\
$c$ & $\{c\}$ & $\{c\}$ & $\{b, c\}$ & $\{0, a, b\}$
\end{tabular}

\begin{tabular}{l|llll}
$\cdot$ & 0 & $a$ & $b$ & $c$ \\
\hline 0 & 0 & $a$ & $b$ & $c$ \\
$a$ & 0 & $a$ & $b$ & $c$ \\
$b$ & 0 & $a$ & $b$ & $c$ \\
$c$ & 0 & $a$ & $b$ & $c$
\end{tabular}

Let $\tau=\{\varnothing, R,\{0, a\}\}$. Then $(R,+, \cdot, \tau)$ is a topological hyper nearrring.

Lemma 1. [2] Let $(R,+, \cdot, \tau)$ be a topological hyper nearring. If $U$ is an open set and a complete part of $R$, then for every $c \in R, c+U$ and $U+c$ are open sets.

Definition 3. 24 A binary relation $\delta$ on $P(X)$ is called a proximity on $X$ if and only if $\delta$ satisfies the following conditions:

(P1) $A \delta B$ implies $B \delta A$,

(P2) $A \delta B$ implies $A \neq \emptyset$,

(P3) $A \cap B \neq \emptyset$ implies $A \delta B$,

(P4) $A \delta(B \cup C)$ if and only if $A \delta B$ or $A \delta C$,

(P5) $A \not \delta B$ implies there exists $E \subseteq X$ such that $A \not \delta E$ and $B \not E^{c}$.

The pair $(X, \delta)$ is called a proximity space. If the sets $A, B \subseteq X$ are $\delta$-related, then we write $A \delta B$, otherwise we write $A \not B B$.

Example 2. Let $A, B \subseteq X$ and $A \delta B$ if and only if $A \neq \emptyset$ and $B \neq \emptyset$. Then $\delta$ is a proximity on $X$.

The following theorem shows a proximity relation $\delta$ on $X$ induces a topology on $X$. 
Theorem 1. [24] If a subset $A$ of a proximity space $(X, \delta)$ is defined to be closed if and only if $x \delta A$ implies $x \in A$, then the collection of complements of all closed sets so defined yields a topology $\tau=\tau(\delta)$ on $X$.

\section{Some Results on tOpOlOGiCAL HyPER NEARRINGS}

In this section, we present some results and properties in topological hyper nearring.

Lemma 2. Let $(R,+, \cdot, \tau)$ be a topological hyper nearring. Then, $0 \in \bigcup_{R \neq U \in \tau} U$.

Proof. If $0 \notin \bigcup_{R \neq U \in \tau} U$, then for every $R \neq U \in \tau, 0 \notin U$. Let $U \in \tau, U \neq \emptyset$ and $0 \neq x \in U$. By the continuity of the mapping + , there exist neighborhoods $V_{1}, V_{2} \in \tau$ of $x$ and 0 , respectively, such that $V_{1}+V_{2} \subseteq U$. Hence, we conclude that $V_{2}=R$ and $V_{1}+R \subseteq U$. Hence, we have $0 \in x+(-x) \subseteq V_{1}+R \subseteq U$ and it is a contradiction. Therefore, we have $0 \in \underset{R \neq U \in \tau}{\bigcup} U$.

Lemma 3. Let $(R,+, \cdot, \tau)$ be a topological hyper nearring such that every open subset of it is a complete part of $R$. Let $\mathcal{U}$ be the system of all neighborhoods of 0 , then for any subset $A$ of $R$,

$$
\bar{A}=\bigcap_{U \in \mathcal{U}}(A+U) .
$$

Proof. Suppose that $x \in \bar{A}$ and $U \in \mathcal{U} . \quad x-U$ is an open neighborhood of $x$, hence we have $x-U \cap A \neq \emptyset$. Thus there exists $a \in A$ such that $a \in x-U$. So, $x \in a+U \subseteq A+U$, for all $U \in \mathcal{U}$. Therefore, $\bar{A} \subseteq \bigcap_{U \in \mathcal{U}}(A+U)$. Now, let $x \in A+U$, for every $U \in \mathcal{U}$ and let $V$ be a neighborhood of $x . x-V$ is a neighborhood of 0 , hence $x \in A+(x-V)$. So, there exist $a \in A$ and $t \in x-V$ such that $x \in a+t$. Thus $a \in x-t \subseteq x+V-x=V$. Then $A \cap V \neq \emptyset$ and this proves that $x \in \bar{A}$ and $\bigcap_{U \in \mathcal{U}}(A+U) \subseteq \overline{\bar{A}}$. Therefore, $\bar{A}=\bigcap_{U \in \mathcal{U}}(A+U)$.

Corollary 1. Let $(R,+, \cdot, \tau)$ be a topological hyper nearring such that every open subset of it is a complete part of $R$ and let $\mathcal{U}$ be the system of all neighborhoods of 0. Then,

(i) $\overline{\{0\}}=\bigcap_{U \in \mathcal{U}} U$;

(ii) For every open set $V$ and every closed set $F$ such that $V \cap \overline{\{0\}} \neq \emptyset$ and $F \cap \overline{\{0\}} \neq \emptyset$, we have $\overline{\{0\}} \subseteq V$ and $\overline{\{0\}} \subseteq F$;

(iii) $\{0\}$ is dense in $R$ if and only if $R$ has trivial topology $\{\emptyset, R\}$.

Proof. (i) It follows immediately from of Lemma 3.

(ii) Let $V$ be open, $V \cap \overline{\{0\}} \neq \emptyset$ and $t \in V \cap \overline{\{0\}}$. $V$ is a neighborhood of $t$ and $t \in \overline{\{0\}}$, thus $V$ is a neighborhood of 0 and by $(i), \overline{\{0\}} \subseteq V$. Now, suppose that 
$F$ is a closed subset and $F \cap \overline{\{0\}} \neq \emptyset$. Then, $\overline{\{0\}} \nsubseteq F^{c} . F^{c}$ is open thus we have $\overline{\{0\}} \cap F^{c}=\emptyset$. Consequently, we get $\overline{\{0\}} \subseteq F$.

(iii) Let $\{0\}$ is dense in $R$ and $U$ be nonempty and open in $R$. Then, $R=\overline{\{0\}}$ and by $(i i) \overline{\{0\}} \subseteq U$. Therefore, $R=U$.

Lemma 4. Let $(R,+, \cdot \tau)$ be a topological hyper nearring such that every open subset of it is a complete part of $R$. Then $\{0\}$ is open if and only if $\tau$ is discrete.

Proof. It is straightforward.

Theorem 2. Let $(R,+, \cdot, \tau)$ be a topological hyper nearring such that every open subset of it is a complete part and $H$ be a normal subhyper group of it. Then $R / H$ is discrete if and only if $H$ is open.

Proof. Suppose that $R / H$ is discrete and $\pi$ is the natural mapping $x \mapsto \pi(x)=$ $H+x$ of $R$ onto $R / H$. Then, the identity, $\pi(0)$ of $R / H$ is an isolated point. So, $\pi^{-1}(\pi(0))=H$ is open of $R$. Now, if $H$ is open, since $\pi$ is open, it follows that $\pi(H)$ is open. Hence the identity $\pi(H)$ of $R / H$ is an isolated point. Therefore, we conclude that $R / H$ is discrete.

Theorem 3. Let $(R,+, \cdot, \tau)$ be a topological hyper nearring such that every open subset of it is a complete part. Then, the following conditions are equivalent:

(1) $R$ is a $T_{0}$ - space;

(2) $\{0\}$ is closed.

Proof. $(1 \Rightarrow 2)$ Let $R$ be a $T_{0}$ - space and let $x \in \overline{\{0\}}$. We prove that $x=0$. If $x \neq 0$, then by (1) there exists an open neighborhood $U$ containing only 0 or $x$, but since $x \in \overline{\{0\}}$, hence $U$ is a neighborhood of 0 , such that $x \notin U$. So, $x \in-U+x$. By Lemma 1. $-U+x$ is an open neighborhood of $\mathrm{x}$, such that $0 \notin-U+x$ (Because if $0 \in-U+x$, then there exists $u \in U$ such that $0 \in-u+x$. So, $x=u+0 \in U)$, this is a contradiction. Thus, $x=0$ and it follows that 0 is closed.

$(2 \Rightarrow 1)$ Let $\{0\}$ be closed and $x, y \in R, x \neq y$. We show that there exist an open neighborhood $U$ containing only $x$ or $y$. If $y=0$, since $\{0\}$ is closed and $x \neq 0$, then $x$ is an interior point of $R \backslash\{0\}$. Hence, there exists a neighborhood $U$ of $x$ such that $0 \notin U$. Now, if $x \neq 0, y \neq 0$ and $x \neq y$, then $0 \notin x-y$. Consequently, by the previous part, for every $t \in x-y$ there exists a neighborhood $U_{t}$ of $t$, such that $0 \notin U_{t}$. We consider $U=\bigcup_{t \in x-y} U_{t}$. Then $x-y \subseteq U$ and $0 \notin U$. Thus $U+y$ is a neighborhood of $x$ such that $y \notin U+y$ (since $0 \notin U)$. Therefore, $R$ is a $T_{0^{-}}$ space.

Let $(X, \tau)$ be a topological space. If $f$ is a arbitrary mapping from $X$ onto $Y$, then consider the family $\tau_{f}=\left\{U: U \subseteq Y, f^{-1}(U) \in \tau\right\}$. Obviously $\tau_{f}$ is a topology on $Y$.

Theorem 4. 25] Let $f:(X, \tau) \rightarrow\left(Y, \tau^{\prime}\right)$ be a continuous function. Then $\tau^{\prime} \leq \tau_{f}$. 
Lemma 5. Let $f: R \rightarrow R^{\prime}$ be a homomorphism of hyper nearrings. Then for every subset $A \subseteq R, f^{-1}(f(A))=\operatorname{kerf}+A$.

Proof. Let $A \subseteq R$ and $t \in f^{-1}(f(A))$. Then $f(t) \in f(A)$ and it follows that there exists $a \in A$ such that $f(t)=f(a)$. Thus $0 \in f(t)-f(a)=f(t-a)$. Hence there exists $x \in t-a$ such that $f(x)=0$. Then $x \in \operatorname{ker} f$. Thus $t \in x+a \subseteq k e r f+A$ and this shows that $f^{-1}(f(A)) \subseteq \operatorname{ker} f+A$. It is obvious that $\operatorname{ker} f+\bar{A} \subseteq f^{-1}(f(A))$. Therefore, $f^{-1}(f(A))=\operatorname{ker} f+A$.

Theorem 5. Let $(R,+, \cdot, \tau)$ and $\left(R^{\prime},+^{\prime}, .^{\prime}, \tau^{\prime}\right)$ be two topological hyper nearring such that every open subset of them is a complete part and $f$ from $R$ onto $R^{\prime}$ be a homomorphism. Then $\left(R^{\prime}, \tau_{f}\right)$ is a topological hyper nearring.

Proof. We should show that $+^{\prime}, .^{\prime}$ and inverse operation are continuous on $\left(R^{\prime}, \tau^{\prime}\right)$. Suppose that $x^{\prime}, y^{\prime} \in R^{\prime}$ and $x^{\prime}+{ }^{\prime} y^{\prime} \subseteq U^{\prime} \in \tau_{f}$. Since $f$ is onto, then there exist $x, y \in R^{\prime}$ such that $f(x)=x^{\prime}$ and $f(y)=y^{\prime}$. Hence $f(x+y)=f(x)+{ }^{\prime} f(y)=$ $x^{\prime}+{ }^{\prime} y^{\prime} \subseteq U^{\prime}$. So, $x+y \subseteq f^{-1}\left(U^{\prime}\right) \in \tau$ (since $\left.U^{\prime} \in \tau_{f}\right)$. Since + is continuous, then there exist neighborhoods $U_{x} \in \tau$ and $U_{y} \in \tau$ of elements $x$ and $y$, respectively, such that $U_{x}+U_{y} \subseteq f^{-1}\left(U^{\prime}\right)$. By Lemmas 1 and $5, f^{-1}\left(f\left(U_{x}\right)\right)=k e r f+{ }^{\prime} U_{x} \in \tau$ and $f^{-1}\left(f\left(U_{y}\right)\right) \in \tau$. Hence $f\left(U_{x}\right) \in \tau_{f}$ and $f\left(U_{y}\right) \in \tau_{f}$. Therefore, we obtain

$$
f\left(U_{x}\right)+{ }^{\prime} f\left(U_{y}\right)=f\left(U_{x}+U_{y}\right) \subseteq f\left(f^{-1}\left(U^{\prime}\right)\right)=U^{\prime} .
$$

This completes the proof.

Theorem 6. Let $f$ from $(R, \tau)$ onto $\left(R^{\prime}, \tau^{\prime}\right)$ be a homomorphism of topological hyper nearrings. Then $f:(R, \tau) \rightarrow\left(R^{\prime}, \tau_{f}\right)$ is continuous and open.

Proof. If $U \in \tau_{f}$, by the definition of $\tau_{f}, f^{-1}(U) \in \tau$. Thus, $f$ is continuous.

Now, let $U$ be an open subset in $R$. Then by Theorem $5 f^{-1}(f(U))=k e r f+U$ is open in $(R, \tau)$. Thus by the definition of $\tau_{f}, f(U) \in \tau_{f}$. This means $f(U)$ is open in $R^{\prime}$. Therefore, $f$ is open.

Let $R$ be a topological hyper nearring, $H$ be normal hyper $R$-subgroup of $R$ and $\pi$ be natural mapping of $R$ onto $R / H$ by $x \mapsto \pi(x)=H+x$. Then, by Theorem $3.30[2]\left(R / H, \tau_{\pi}\right)$ is a topological hyper nearring. It is called the quotient space of topological hyper nearring $R$ that we showed $\tau_{\pi}$ by $\bar{\tau}$ in 2 .

Theorem 7. Let $R$ be a $T_{0}$-topological hyper nearring such that every open subset of it is a complete part of $R$ and $H$ be a discrete subhypergroup of $R$. Then $H$ is closed.

Proof. Let $x \in \bar{H}$. Since $H$ is a discrete subhypergroup of $R$, then $0 \in H$ and there exists an open neighborhood $V$ of 0 such that $V \cap H=\{0\}$. By Lemma 1. $x-V$ is an open neighborhood of $x$. Therefore, $x-V \cap H \neq \emptyset$ (because $x \in \bar{H}$ ). Hence there exists $h \in H$ such that $h \in x-V$ and $h \in x-v$, for some $v \in V$. Thus $v \in-h+x \subseteq V \cap \bar{H} \subseteq \overline{V \cap H}$ (let $t \in V \cap \bar{H}$ and $U_{t}$ is a neighborhood of t. $U_{t} \cap V$ is an open neighborhood of $t$ and since $t \in \bar{H}$, then $\left(U_{t} \cap V\right) \cap H \neq \emptyset$ 
and $U_{t} \cap(V \cap H) \neq \emptyset$. It follows that $t \in \overline{V \cap H}$ and $\left.V \cap \bar{H} \subseteq \overline{V \cap H}\right)$. Thus $v \in \overline{V \cap H}=\overline{\{0\}}=\{0\}$ (by Theorem 3 ) and it follows that $x=h \in H$ and $H$ is closed.

Theorem 8. Let $R$ be a topological hyper nearring and $H$ a dense subhypergroup of $R$. If $V$ is a neighborhood of 0 in $H$, then $\bar{V}$ is a neighborhood of 0 of $R$.

Proof. Since $V$ is a neighborhood of 0 in $H$, it follows that there exists an open neighborhood $U$ of 0 in $R$ such that $U \cap H \subseteq V$. Hence, we obtain $U=U \cap G=$ $U \cap \bar{H} \subseteq \overline{U \cap H} \subseteq \bar{V}$. Therefore, 0 is an interior point $\bar{V}$ and $\bar{V}$ is open in $R$.

\section{TOPOLOGICAL HYPER NEARRING DERIVED FROM A PROXIMITY SPACE}

In this section, we define a proximity relation on an arbitrary hyper nearring and prove that every hyper nearring with topology whose is induced by this proximity relation is a topological hyper nearring. Also, we show that every topological hyper nearring is a proximity space.

Theorem 9. Let $(R,+, \cdot)$ be a hyper nearring, $N$ be a normal subhypergroup of $R$ and $A, B \subseteq R$. We define $A \delta B$ if and only if there exist $a \in A$ and $b \in B$ such that $-b+a \subseteq N$, then $(R, \delta)$ is a proximity space.

Proof. ( $\left.P_{1}\right)$ Suppose that $A \delta B$. Then, there exist $a \in A$ and $b \in B$ such that $-b+a \subseteq N$. So, we get $-a+b \subseteq-N=N$. Therefore, $B \delta A$.

$\left(P_{2}\right)$ It is obvious.

$\left(P_{3}\right)$ Let there exists $x \in A \cap B \neq \emptyset$. Then $-x+x \subseteq-x+N+x \subseteq N$. So, we conclude that $A \delta B$.

$\left(P_{4}\right)$ It is straightforward.

$\left(P_{5}\right)$ Let $A \not \delta B$ and $E:=B+N$. If $A \delta E=B+N$, then there exist $a \in A$ and $b \in B$ such that $-(b+N)+a \subseteq N$. Therefore, $-N-b+a \subseteq N$ and this implies that $-b+a \subseteq N+N \subseteq N$. Thus, $A \delta B$ and it is a contradiction. Hence $A \not \delta E$. Also, $B \not \delta E^{c}$. If $B \delta E^{c}$, then there exist $b \in B$ and $x \in(B+N)^{c}$ such that $-x+b \subseteq N$. Therefore, $x \in b+N \subseteq B+N$ and it is a contradiction.

Theorem 10. In the proximity space $(R, \delta)$ that $(R,+, \cdot)$ is a hyper nearring and $\delta$ is defined relation in Theorem g, the set $\beta=\{x+N: x \in R\}$ is a base for the topology $\tau=\tau(\delta)$.

Proof. Let $U$ be an open subset of $R$ and let $y \in U$. We should show that $y+N \subseteq U$. Let $t \notin U$, then $t \in U^{c}$ and $t \delta U^{c}$ (since $U^{c}$ is closed). $-y+t \subseteq-y+y+N \subseteq$ $-y+N+y \subseteq N$. Hence $t \delta y$ and by $(P 4), y \delta U^{c}$. Thus $y \in U^{c}$ and it is a contradiction. This implies that $\beta$ is a base for the topology $\tau(\delta)$.

Lemma 6. The normal subhypergroup $N$ of $R$ is a clopen set in the topology $\tau(\delta)$ is defined in Theorem 10. 
Proof. By Theorem 10, $N$ is open. Now, let $x \delta N$, for $x \in R$. Then there exists $n \in N$ such that $-n+x \subseteq N$. Therefore $x \in n-n+x \subseteq n+N=N$. Thus $N$ is a closed subset in $R$.

Theorem 11. Let $(R,+, \cdot)$ be a hyper nearring, the normal subhypergroup $N$ be a complete part of $R$ and the relation $\delta$ is defined in Theorem 9 . Then the system $(R,+, \cdot, \tau(\delta))$ is a topological hyper nearring.

Proof. We should show that + , and inverse operation are continuous. Suppose that $U$ is an open subset of $R$ such that $x+y \subseteq U$, for $x, y \in R$. Then by Theorem 10. there exists $t \in R$ such that $x+y \subseteq t+N \subseteq U$. Therefore, $x+N$ and $y+N$ are neighborhoods of $x$ and $y$ such that $(x+N)+(y+N)=x+y+N \subseteq$ $t+N+N=t+N \subseteq U$. Thus + is continuous on $R$. Now, Suppose that $U$ is an open neighborhood of $-x$. By Theorem 10 , there exists $t \in R$ such that $-x \in t+N \subseteq U$. Therefore, $x \in-N-t=-t+N$. Hence $-t+N$ is a neighborhoods of $x$ and $-(-t+N)=-N+t=N+t=t+N \subseteq U$. This proves that inverse operation is continuous. Now, we show that - is continuous. Suppose that $U$ is an open subset of $R$ such that $x \cdot y \in U$, for $x, y \in R$. Then there exist $t \in R$ such that $x \cdot y \in t+N \subseteq U$ ( by Theorem10p. $x+N$ and $y+N$ are neighborhoods of $x$ and $y$ such that $(x+N) \cdot(y+N) \subseteq x \cdot y+N(N$ is a complete part of $R$, then $x \cdot y+N$ is a complete part of $R$. Hence $(x+N) \cdot(y+N) \subseteq x \cdot y+N)$. So, $(x+N) \cdot(y+N) \subseteq x \cdot y+N \subseteq t+N+N=t+N \subseteq U$. Thus $\cdot$ is continuous on $R$.

Example 3 . Let $R=\{0, a, b\}$ be a set with a hyperoperation + and a binary operation $\cdot$ as follows:

\begin{tabular}{c|ccc}
+ & 0 & $a$ & $b$ \\
\hline 0 & $\{0\}$ & $\{a\}$ & $\{b\}$ \\
$a$ & $\{a\}$ & $\{0\}$ & $\{b\}$ \\
$b$ & $\{b\}$ & $\{b\}$ & $\{0, a\}$
\end{tabular}

\begin{tabular}{l|lll}
$\cdot$ & 0 & $a$ & $b$ \\
\hline 0 & 0 & $a$ & $b$ \\
$a$ & 0 & $a$ & $b$ \\
$b$ & 0 & $a$ & $b$
\end{tabular}

Then, $(R,+, \cdot)$ is a hyper nearring. We consider a normal subhyperring $N=$ $\{0, a\}$ of $R$ and define:

$A \delta B$ if and only if there exist $a \in A$ and $b \in B$ such that $-b+a \subseteq N$.

Therefore, $\tau(\delta)=\{\varnothing,\{0, a, b\},\{0, a\},\{b\}\}$. Simply, we can show that $(R,+, \cdot, \tau(\delta))$ is a topological hyper nearring.

The following theorem, show that every topological hyper nearring is a proximity space. 
Theorem 12. Let $(R,+, \cdot, \tau)$ be a topological hyper nearring such that every open subset of it is a complete part of $R$. Then there exists a proximity relation $\delta$ such that $(R, \delta)$ is a proximity space.

Proof. Let $\mathcal{U}$ be the system of symmetric neighborhoods at 0 , for every $A, B \subseteq R$ and $V \in \mathcal{U}$. We define

$A \delta B$ if and only if $A \cap B+V \neq \emptyset$.

Now, we show that $\delta$ is a proximity relation.

$\left(P_{1}\right)$ Suppose that $A \delta B$. Then, there exist $a \in A$ and $b \in B$ such that $a \in b+V$. Hence $b \in a-V=a+V \subseteq A+V$. Therefore, $B \delta A$.

$\left(P_{2}\right)$ It is obvious.

$\left(P_{3}\right)$ Let $A \cap B \neq \emptyset$. Then, there exists $x \in A \cap B$. Therefore, $x \in A \cap B+V \neq \emptyset$. Thus $A \delta B$.

$\left(P_{4}\right)$ It is straightforward..

$\left(P_{5}\right)$ Let $A / \delta B$ and $E:=B+V$. If $A \delta B+V$, then $A \cap(B+V)+V \neq \emptyset$. Therefore $A \cap B+V \neq \emptyset$ (since $V$ is a complete part of $R$, then $V+V \subseteq V$ ) and this proves that $A \delta B$, that it is a contradiction. Hence $A \not \delta E$. Also, if $B \delta E^{c}$, it follows that $B \cap(B+V)^{c}+V \neq \emptyset$. Hence there exist $b \in B, x \in(B+V)^{c}$ and $v \in V$ such that $b \in x+v$. Thus $x \in b-v \subseteq B+V$ and it is a contradiction. Therefore, $B \not \delta E^{c}$.

\section{Conclusion}

In this paper we expressed the relationship between two important subjects: algebraic hyperstructures and topology. We studied several characteristics of topological hyper nearrings and in the following, we related them to proximity spaces.

\section{REFERENCES}

[1] Ameri, R., Topological (transposition) hypergroups, Italian Journal of Pure and Applied Mathematics, (13) (2003), 181-186.

[2] Borhani-Nejad, S., Davvaz, B., Topological hyper nearrings, submitted.

[3] Corsini, P., Prolegomena of Hypergroup Theory, Second ed., Aviani Editore, Tricesimo, Italy, 1993.

[4] Corsini, P., Leoreanu, V., Applications of Hypergroup Theory, Kluwer Academic Publishers, 2003.

[5] Dasic, V., Hypernearrings, Fourth Int. Congress on Algebraic Hyperstructures and Applications (AHA 1990), World Scientific, (1991), 75-85.

[6] Davvaz, B., Hypernearrings and weak hypernearrings, 11th Algebra Seminar of Iranian Math. Soc. Isfahan University of Technology, Isfahan, October 27-29, (1999), 68-78.

[7] Davvaz, B., Polygroup Theory and Related Systems, World Scientific Publishing Co. Pte. Ltd., Hackensack, NJ, 2013.

[8] Davvaz, B., Semihypergroup Theory, Elsevier, 2016.

[9] Davvaz, B., Leoreanu-Fotea, V., Hyperring Theory and Applications, International Academic Press, 115, Palm Harber, USA, 2007.

[10] Efremovč, V.A., The geometry of proximity, Mat. Sb., 31 (1952), 189-200.

[11] Efremovič, V.A., Infinitesimal spaces, Doklady Akademii Nauk SSSR (N.S.), (in Russian), 76 (1951), 341-343. 
[12] Freni, D., A note on the core of a hypergroup and the transitive closure $\beta^{*}$ of $\beta$, Riv. Mat. Pura Appl., 8 (1991), 153-156.

[13] Gontineac, V.M., On hypernear-rings and H-hypergroups, Fifth Int. Congress on Algebraic Hyperstructures and Applications (AHA 1993), Hadronic Press, Inc., USA, (1994), 171-179.

[14] Heidari, D., Davvaz, B., Modarres, S.M.A., Topological hypergroups in the sense of Marty, Comm. algebra, 42 (2014), 4712-4721.

[15] Heidari, D., Davvaz, B., Modarres, S.M.S., Topological polygroups, Bull. Malays. Math. Sci. Soc., 3942 (2016), 707-721.

[16] Hošková-Mayerová, S., Topological hypergroupoids, Comput. Math. Appl., 64(9) (2012), $2845-2849$.

[17] Jancic-Ras̆ovic S., Cristea, I., Division hypernear-rings, An. Stiint. Univ. "Ovidius" Constanta Ser. Mat., 26(3) (2018), 109-126.

[18] Jancic-Ras̆ovic S., Cristea, I., Hypernear-rings with a defect of distributivity, Filomat, 32(4) (2018), 1133-1149.

[19] Kim, K.H., Davvaz, B., Roh, E.H., On Hyper R-subgroups of hypernear-rings, Scientiae Mathematicae Japonicae, Online (2007), 649-656.

[20] Koskas, M., Groupoides, demi-hypergroupes et hypergroupes, J. Math. Pure Appl., 49(9) (1970), 155-192.

[21] Munkres, J.R., Topology, Prentice Hall, Upper Saddle River, NJ, 2000.

[22] Marty, F., Sur une generalization de la notion de group, 8th congress Math. Scandinaves, Stockholm, (1934), 45-49.

[23] Mittas, J., Hypergroups canoniques, Math. Balkanica, 2 (1972), 165-179.

[24] Naimpally S.A., Warrack, B.D., Proximity Spaces, Cambridge Tract, 1970.

[25] Ursul, M., Topological rings satisfying compactness conditions, Springer Science Business Media (2002), vol 549. 